\title{
Comparative Adherence of Granulocytes to Endothelial Monolayers and Nylon Fiber
}

\author{
Rob Roy MacGregor, Edward J. Macarak, and Nicholas A. Kefalides, \\ Infectious Diseases Section, Department of Medicine, and the Connective \\ Tissue Research Institute, University of Pennsylvania School of Medicine, \\ Philadelphia, Pennsylvania 19104
}

A B S TRACT Adherence of granulocytes to tissue culture monolayers of endothelium averaged 26.2 $\pm 1.3 \% \mathrm{SEM}$, which was similar to their adherence on 50 -mg nylon fiber columns $(27.7 \pm 3.6 \%)$. In contrast, adherence to epithelial cells, fibroblasts, kidney cells, and plastic Petri dishes without monolayers was only $12.4,9.9,11.1$, and $4.3 \%$, respectively. Cyclic nucleotides and adherence-modifying plasma factors induced changes of adherence to endothelium similar to those in nylon fiber columns. Adherence of granulocytes in whole blood was the same as for purified granulocytes in Hanks' balanced salt solution.

Exposure of endothelial monolayers to $0.18 \%$ trypsin for $10 \mathrm{~min}$ reduced subsequent granulocyte adherence to $25.2 \%$ of control values. Incubation of trypsin-treated monolayers with nutrient medium for $4 \mathrm{~h}$ did not improve adherence, but values returned to normal or above by $24 \mathrm{~h}$, with or without serum proteins present in the nutrient medium.

The similarity of granulocyte adherence to nylon fiber and to endothelial monolayers in vitro suggests that results with the nylon fiber assay reflect in vivo granulocyte-endothelium interaction. Furthermore, the endothelial monolayer offers a new model for studying this cell-cell relationship in vitro.

\section{INTRODUCTION}

The adherence of granulocytes to vascular endothelium is necessary before their diapedesis into the tissues. In 1961, Garvin used columns packed with glass beads to measure granulocyte adherence (GA) ${ }^{1}$

This work was presented in part at the American Federation for Clinical Research eastern meeting, Boston, Mass., January 1977.

Received for publication 1 August 1977 and in revised form 28 October 1977.

${ }^{1}$ Abbreviation used in this paper: GA, granulocyte adherence. in vitro, and defined some characteristics of the adherence phenomenon (1). Recently, we confirmed his findings, using a simpler in vitro adherence assay which utilizes spun nylon fiber columns and small volumes of heparinized whole blood (2). Clinical studies with the nylon fiber assay have shown adherence to be increased in conditions of granulocyte margination and rapid egress from the intravascular compartment $(3,4)$, and to be decreased in conditions of poor granulocyte delivery such as during treatment with steroids or other anti-inflammatory agents $(2,5)$. Thus, GA values obtained from the nylon fiber assay appear to be directly related to the ease of cell movement out of the vascular compartment.

Despite these clinical correlations, questions remain regarding the appropriateness of applying data derived from granulocyte adherence to an inert nylon fiber surface in vitro to the phenomenon of granulocyte-endothelial cell interaction in vivo. The development of effective techniques for culturing endothelial cells (6-9) now offers an opportunity to study the adherence of granulocytes to endothelial monolayers in vitro, and to compare adherence characteristics on monolayers with those in nylon fiber columns.

\section{METHODS}

The nylon fiber assay for GA has been described in detail in an earlier publication (2). Carefully weighed amounts of spun nylon fiber are packed into Pasteur pipettes to a column length of exactly $15 \mathrm{~mm}$. 1-ml volumes of heparinized whole blood are applied to the top of triplicate columns and allowed to flow through by gravity at $37^{\circ} \mathrm{C}$. Comparison of the pre- and postcolumn granulocyte counts permits calculation of the percent of granulocytes adhering to the column. Results of the triplicate columns are averaged for each blood specimen and expressed as the percent granulocyte adherence. Values for the triplicate columns fall within $15 \% \pm$ the mean value. Because the percentage of adherence found on the endothelial monolayers was low, columns packed with $50 \mathrm{mg}$ of fiber, which result in a mean adherence of $27.7 \pm 3.6 \%$ in normals, were used in all experiments comparing adherence on endothelium to that on nylon. 
All experiments were repeated a minimum of three times and the mean values were compared by the Student's $t$ test. All cell counts were performed with an electronic particle counter.

Cultures of human endothelial cells were established from human umbilical cord veins. Umbilical cords were obtained soon after delivery and placed in Medium 199 (Kansas City Biological, Lenexa, Kans.). Umbilical vein endothelial cells were isolated using the method of Gimbrone et al. (8) and grown in Medium 199 as modified by Lewis et al. (6). The Medium 199 was supplemented with $20 \%$ fetal bovine serum (Rehatuin, Reheis Chemical Co., Berkeley Heights, N. J.) and antibiotics (gentamicin, $50 \mu \mathrm{g} / \mathrm{ml}$ and amphotericin B, $2.5 \mu \mathrm{g} / \mathrm{ml}$ ). All endothelial cells used in this study were primary monolayers, 6-9 days of age, and three to five generations after isolation. All monolayers were confluent. In addition, Petri dishes containing monolayers of human lung fibroblasts (WI-38), human epithelium (HEP-2), and rabbit kidney cells (RK-13), grown until confluent in Eagle's minimal essential medium supplemented with $7 \%$ fetal calf serum, were supplied by Dr. Harvey Friedman, Viral Diagnostic Laboratory, Children's Hospital of Philadelphia. Total cell protein content was measured for each cell monolayer by the Lowry technique, to determine whether cell concentration was roughly analogous for each cell type.

In most experiments, heparinized $(5 \mathrm{U} / \mathrm{ml})$ venous whole blood (leukocyte counts $5-10,00(0) / \mathrm{mm}^{3}$ ) from normal donors was used to measure GA. Medium 199 was decanted from the tissue cultures and $1 \mathrm{ml}$ of blood was added to triplicate plates, just covering the $35-\mathrm{mm}$ diameter surfaces. The tissue culture-blood overlay was incubated at $37^{\circ} \mathrm{C}$ and $100 \%$ humidity for $15 \mathrm{~min}$ without agitation, and then the blood was aspirated. Comparison of the granulocyte counts before and after incubation allowed calculation of the percentage of granulocytes adhering to the monolayer. In some experiments, a pure granulocyte suspension was prepared by Ficoll-Hypaque (Ficoll, Pharmacia Fine Chemicals Inc., Hypaque, Winthrop Laboratories) density gradient sedimentation using the technique of Boyum (10). After separation, the granulocytes were washed three times in modified Hanks' solution and suspended in Hanks' balanced salt solution at a concentration of $5-10 \times 10^{6}$ cells $/ \mathrm{ml}$. The suspension was layered over the monolayer, incubated, and the percent GA calculated as above. In experiments evaluating the effect of adherence-modifying plasma factors, heparinized whole blood was centrifuged at $80 \mathrm{~g}$ for $10 \mathrm{~min}$ at $4^{\circ} \mathrm{C}$, the cell button was washed three times with modified Hanks' solution, and then resuspended volume for volume in either normal ABO-compatible plasma or in plasma containing the factor being studied. Previous studies have shown that normal cells can be separated and resuspended in this manner without affecting their adherence $(3,5)$. Plasma containing the factor which augments granulocyte adherence (3) was obtained from patients with acute inflammatory diseases. Plasma containing the factor which inhibits adherence (5) was obtained from normal volunteers $4 \mathrm{~h}$ after the ingestion of $40 \mathrm{mg}$ prednisone. All plasma was stored at $-70^{\circ} \mathrm{C}$ until used.

Dibutryl cyclic AMP and 8-bromo cyclic GMP (Sigma Chemical Co., St. Louis, Mo.) were prepared in $0.001 \mathrm{mM}$ stock solutions in normal saline and used within $1 \mathrm{~h}$ of preparation.

In experiments evaluating the effect of trypsin treatment of the endothelial monolayers on GA, a $2.5 \%$ stock solution of trypsin (Grand Island Biological Co., Grand Island, N. Y.) was prepared in Earle's balanced salt solution and kept at $-20^{\circ} \mathrm{C}$ until used. At the time of experiment, the nutrient medium was decanted from the endothelial mono- layers and trypsin solution was added in concentrations from 0.06 to $0.18 \%$. Then the monolayers were incubated for $10 \mathrm{~min}$ at $37^{\circ} \mathrm{C}$, and the trypsin inhibitor (Aldrich Chemical Co. Inc., Milwaukee, Wis.), in Earle's balanced salt solution, was added to the monolayer and incubated for $30 \mathrm{~min}$. After removal of the trypsin inhibitor, the monolayers were covered with modified medium 199 plus $20 \%$ fetal calf serum and incubated at $37^{\circ} \mathrm{C}$ until time for the determination of granulocyte adherence (immediately after trypsinization, 4 , or $24 \mathrm{~h}$ later). In some experiments, the fetal calf serum was omitted to assess its importance to the monolayer's regeneration of normal granulocyte adherence properties.

\section{RESULTS}

The mean adherence of granulocytes in heparinized whole blood to human endothelial monolayers was $26.2 \pm 1.3 \%$ SEM in 10 experiments, compared to adherence of $4.3 \pm 0.6 \%$ in Petri dishes not containing a monolayer (Fig. 1). Adherence on triplicate monolayers varied $<15 \% \pm$ the mean value. In contrast to granulocytes, few lymphocytes adhered to the monolayer.

To determine if the adherence results simply represented settling of the cells, other monolayers were overlayered with blood and either incubated without motion, or gently agitated every $30 \mathrm{~s}$ for $15 \mathrm{~min}$. The stationary monolayers had $20.1 \pm 0.8 \%$ granulocyte adherence, and the agitated monolayers had 25.7 $\pm 3.0 \%$.

Granulocyte adherence to other cell types in tissue culture was greater than to the empty Petri dishes though less than to the endothelial monolayer: 12.4

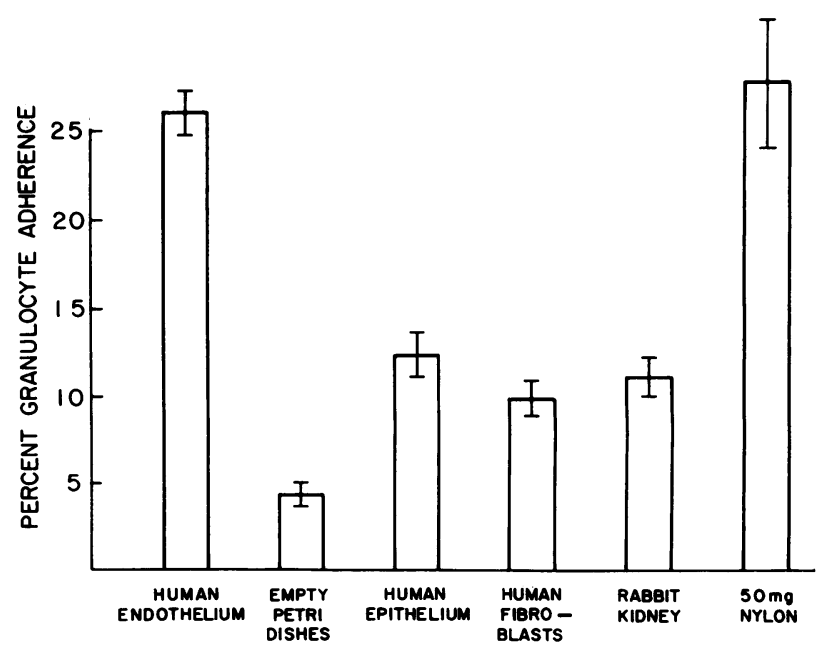

FIGURE 1 Granulocyte adherence to different types of cell monolayers and to nylon fiber columns. Isobars represent mean values for a minimum of three experiments, brackets \pm SEM. Adherence to epithelium, fibroblasts, and kidney cells was greater than to empty Petri dishes, but less than to endothelium or nylon fiber $(P<0.05$, Student's $T$ test $)$. 
$\pm 1.3 \%$ on human epithelial cells, $9.9 \pm 1.0 \%$ on human lung fibroblasts, and $11.1 \pm 1.1 \%$ on rabbit kidney cells. Adherence to the other cell types was significantly less than adherence to endothelium in each case $(P<0.05$, Student's $t$ test). Total protein content for each monolayer was $127 \pm 28.3 \mu \mathrm{g} / \mathrm{ml}$ SEM for endothelium, $304 \pm 10.6$ for epithelium, $336 \pm 69.7$ for fibroblasts, and $551 \pm 79.8$ for rabbit kidney. Thus, the greater adherence to endothelial monolayers does not seem to result from more cell surface area present with this cell type than with the other monolayers. The fact that endothelium had the lowest protein content of the four cell types probably results from the very flat profile and thus less cytoplasm for these cells compared to fibroblasts or kidney cells. When a pure preparation of granulocytes was suspended in Hanks' balanced salt solution, a similar differential adherence was noted between different cell types, whereas mean adherence to endothelium was $46.4 \pm 7.8 \%$ SEM, adherence to epithelium was $17.6 \pm 1.4 \%$, to fibroblasts $21.6 \pm 3.2 \%$ and to rabbit kidney cells $17.1 \pm 3.8 \%$. Adherence to nylon columns was $42.7 \pm 3.1 \%$. Adherence of the pure granulocyte suspension was significantly greater to endothelium than to any of the other cell types $(P<0.05$, Student's $t$ test $)$. Because adherence values on the endothelial monolayers were very close to those reported previously (2) for normal blood on 50-mg ("low adherence") nylon columns (Fig. 1), we used such columns in the subsequent experiments comparing granulocyte adherence on endothelial monolayers with that on nylon fiber columns.

To determine whether granulocytes which fail to adhere the first time are capable of adherence if reincubated with a fresh monolayer, experiments were performed in which the same aliquot of whole blood was incubated sequentially onto two different endothelial monolayers. Mean adherence to the first monolayers was $20.1 \pm 0.8 \% \mathrm{SEM}$, and to the second monolayers was $17.5 \pm 3.4 \%$. Thus, adherence appears to be a random phenomenon rather than representing a subgroup capable of adherence within the total population of granulocytes.

Fig. 2 illustrates comparative granulocyte adherence to the two surfaces under conditions previously shown to modify adherence to nylon fiber. Plasma from patients with acute inflammation, which contains an adherence-augmenting factor, caused a significant increase in granulocyte adherence measured with either surface: from $26.2 \pm 1.3$ to $47.4 \pm 4.1 \%$ on endothelium, and from $27.7 \pm 3.6$ to $54.2 \pm 4.7 \%$ on nylon $(P<0.05$ for each, Student's $t$ test). Plasma from patients treated with prednisone, containing the factor which inhibits adherence, decreased the granulocyte adherence to both surfaces: $20.5 \pm 1.9$ and $19.6 \pm 1.4 \%$, respectively. Addition of $0.01 \mathrm{mM}$ cyclic GMP or cyclic AMP to

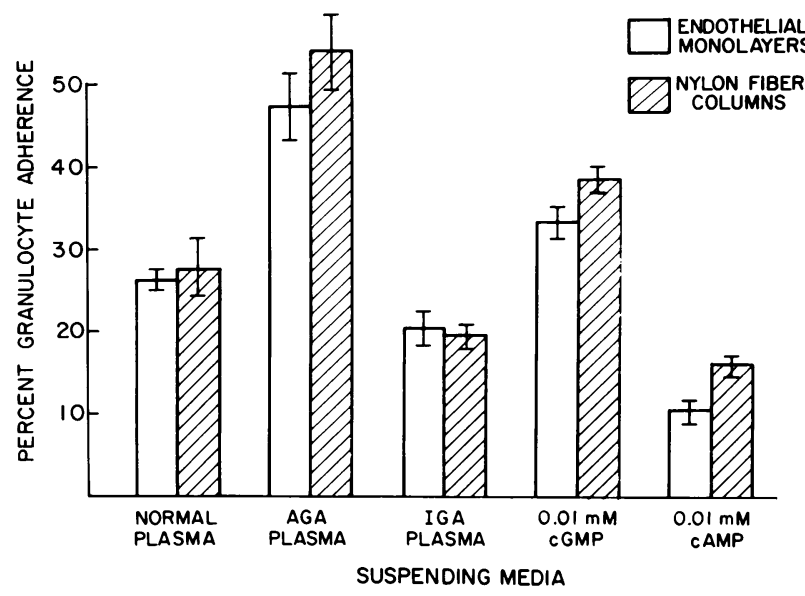

FIGURE 2 Adherence of granulocytes suspended in various media to endothelium and nylon fiber. Isobars represent mean values for a minimum of three experiments, brackets \pm SEM. AGA factor plasma is from patients with augmented granulocyte adherence associated with inflammation, and IGA factor plasma is from patients with inhibited granulocyte adherence after prednisone administration. Cyclic nucleotides were added to normal plasma in the last two experiments. On both surfaces, adherence of granulocytes suspended in normal plasma differed significantly from adherence in the other media $(P<0.05$, Student's $t$ test $)$.

heparinized whole blood, respectively known to increase and decrease adherence to nylon (11), induced similar changes on both surfaces (significantly different from normal, $P<0.05$, Student's $t$ test, but not significantly different between the two surfaces). Addition of $0.01 \mu \mathrm{M}$ propranolol to whole blood raised GA to $38.9 \pm 1.7 \%$ on endothelium, and $34.9 \pm 1.0 \%$ on nylon (data not shown); $0.01 \mu \mathrm{M}$ atropine reduced adherence to $9.8 \pm 3.2 \%$ on endothelium, and 9.1 $\pm 1.6 \%$ on nylon. All changes were significantly different from normal, $P<0.05$, Student's $t$ test.

To determine whether GA to endothelial monolayers requires other blood cell types, platelets, or plasma proteins, a pure suspension of $5-10 \times 10^{6}$ granulocytes $/ \mathrm{ml}$ in Hanks' balanced salt solution was prepared by Hypaque-Ficoll density sedimentation. No erythrocytes remained. The adherence of this suspension was measured on the endothelial monolayer with and without the addition of $0.01 \mathrm{mM}$ cA.MP or cGMP. Adherence of the pure granulocytes in Hanks' balanced salt solution averaged $47.9 \pm 2.7 \%$ SEM for three experiments. Addition of $0.01 \mathrm{mM}$ CAMP reduced adherence to $23.9 \pm 5.6 \%$, whereas cGMP increased it to $69.6 \pm 5.5 \%$; both changes were significantly different from normal $(P<0.05$, Student's $t$ test). In contrast, suspending the purified granulocytes in normal plasma led to the same level of adherence seen when the cells were in Hanks' balanced salt solution, $46.5 \pm 4.7 \%$. Thus, plasma proteins are not re- 


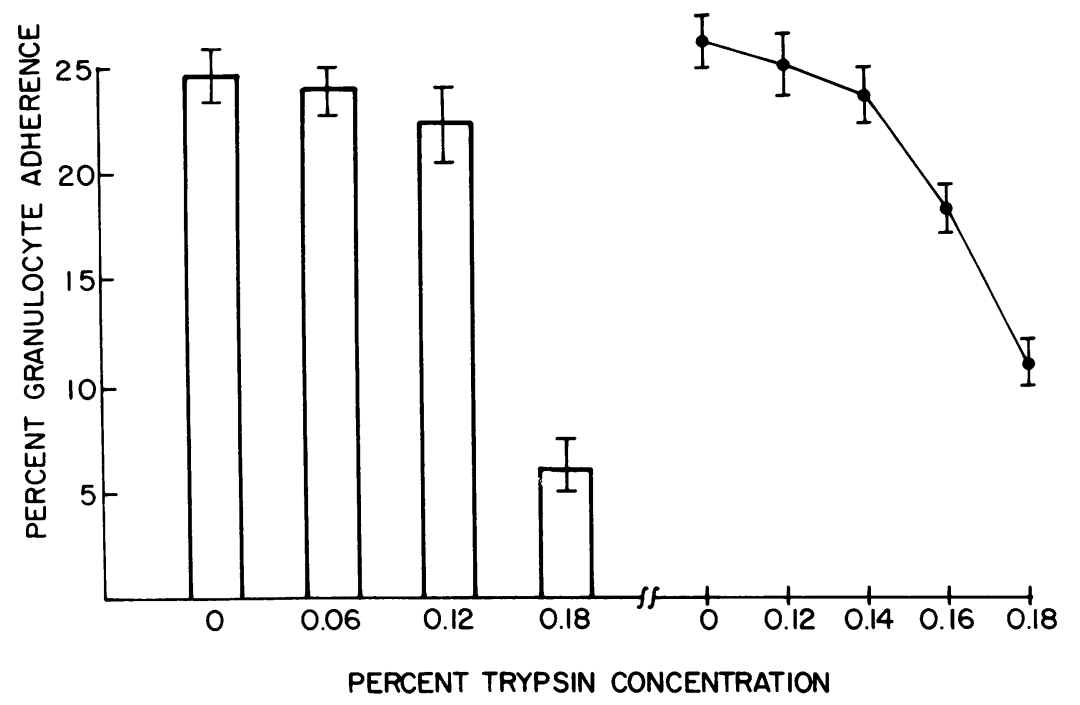

FIGURE 3 Effect of trypsin treatment of the endothelial monolayers on subsequent granulocyte adherence. (A) Isobars represent mean values for a minimum of three experiments, brackets \pm SEM. Significant inhibition of adherence occurred only after exposure of the monolayers to $0.18 \%$ trypsin $(P<0.001$, Student's $t$ test). (B) $0.02 \%$ increments were used between 0.12 and $0.18 \%$; significant inhibition occurred only with $0.16 \%(P<0.02)$ and with $0.18 \%$ trypsin $(P<0.001$, Student's $t$ test).

quired for granulocyte adherence to endothelial cells, nor are other cell types necessary either for normal adherence or for the modification of adherence by cyclic nucleotides.

The intensity of granulocyte adherence to trypsinized endothelial monolayers is shown in Fig. 3. No significant change in adherence was noted until the trypsin concentration was raised to $0.18 \%$. After a 10-min exposure to this concentration, the monolayer could only support a mean granulocyte adherence of $6.2 \pm 1.3 \%$, as compared to $24.6 \pm 1.4 \%$ with the untrypsinized cells $(P<0.001$, Student's $t$ test). The viability of monolayer cells after exposure to $0.18 \%$ trypsin was $96.1 \%$ by trypan blue exclusion, and microscopic examination demonstrated that the monolayer remained confluent and appeared normal. To define the critical concentration of trypsin necessary to inhibit GA to the monolayer, the studies were repeated, with $0.02 \%$ increments in trypsin concentration between 0.12 and $0.18 \%$ (Fig. 3). Only the adherence after 0.16 and $0.18 \%$ exposures was significantly less than on untreated monolayers $(P<0.02$ and 0.001 Student's $t$ test); changes induced by lower concentrations were not significant.

The reversibility of the trypsin effect on GA is shown in Fig. 4. Fig. 4A first shows that incubating the endothelial monolayer with soybean trypsin inhibitor alone has no effect on subsequent GA. Second, treating the monolayers for $10 \mathrm{~min}$ with $0.18 \%$ trypsin, followed by a 30 -min incubation with trypsin inhibitor, led to a significant decrease in subsequent GA $(P$
$<0.05$, Student's $t$ test). Finally, overlaying the trypsinized monolayers with the standard nutrient medium for $4 \mathrm{~h}$ did not improve subsequent GA, but incubation for $24 \mathrm{~h}$ after trypsinization completely

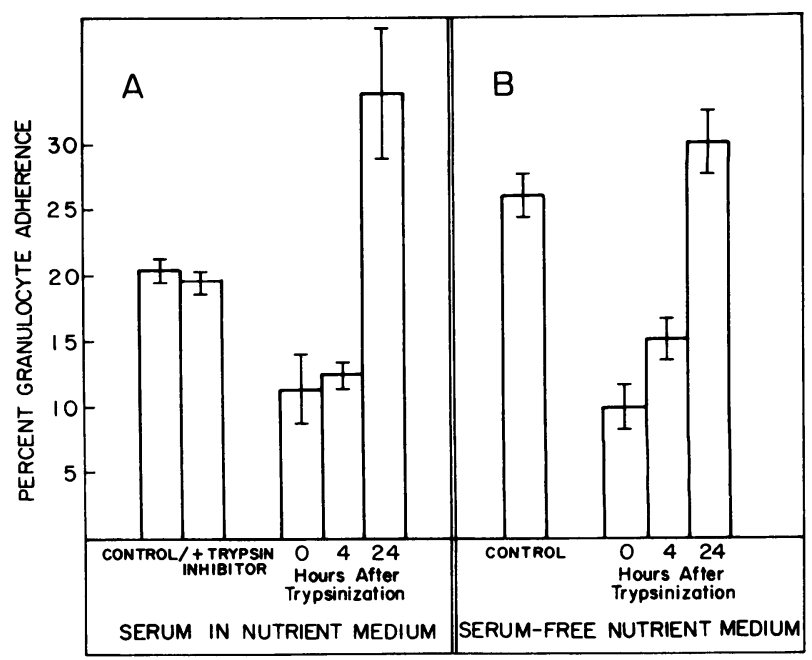

FIGURE 4 Reversibility of trypsin-induced inhibition of granulocyte adherence to endothelial monolayers. Isobars represent the mean of at least three experiments, brackets \pm SEM. In the experiments displayed in A, $20 \%$ fetal calf serum was present in the nutrient medium covering the monolayers after their exposure to $0.18 \%$ trypsin; in B experiments, serum was omitted. Adherence was still low $4 \mathrm{~h}$ after trypsinization but returned to normal or above normal under both conditions. 
corrected the inhibited adherence. Although the adherence value after $24 \mathrm{~h}$ was well above control, the difference was not significant $(P<0.1,>0.05$, Student's $t$ test). The experiments were repeated with $10 \%$ fetal calf serum excluded from the post-trypsinization nutient medium Fig. 4B. Correction of the impaired GA was complete after the 24-h incubation; thus serum proteins are not required in the nutrient medium for trypsinized endothelial monolayers to regenerate the properties necessary for normal GA.

\section{DISCUSSION}

The development of techniques for establishing in vitro cultures of human endothelial cells $(6-8)$ has allowed the study of many aspects of endothelial function inaccessible up to now. Several characteristics document that the monolayers are composed of endothelium: first, the tissue culture morphology shows plate-like cells arranged in a tight monolayer, without any overgrowth. In contrast, fibroblasts appear as spindle-shaped cells, and smooth muscle cells overgrow one another, giving a clumped appearance to the culture (12). Second, the presence of rod-shaped organelles called Weibel-Palade bodies is unique to endothelium (12). Third, immunofluorescence microscopy demonstrates that antihemophilic factor is present in the cells of the endothelial monolayer, in contrast, to fibroblasts and smooth muscle cells (13). These three characteristics were demonstrated by the monolayers used in the present studies.

The greater adherence of granulocytes to monolayers of all cell types tested than to the empty plastic Petri dishes is consistent with the well-known attribute of metazoan cells to adhere (14). However, adherence to the endothelium was more than twice as great as to the other cell lines, suggesting a specific adaptation between granulocytes and endothelial cells for attachment. Such an adaptation or receptor would facilitate granulocyte delivery, because they must attach to the endothelial surface before diapedesis into the tissues (15).

The parallelism of adherence values found between 5()$-m g$ columns and the endothelial monolayers was striking. In every situation where adherence to the nylon fiber columns had been previously reported to be altered, similar changes were noted with the endothelial monolayers. Although concern has been expressed regarding the extrapolation of data derived from adherence to nylon fiber to the more physiological event of granulocyte adherence to endothelium, present studies indicate that the adherence characteristics of the two surfaces are similar. Thus, adherence measured with the in vitro nylon fiber assay appears to reflect in vivo granulocyte-endothelium interactions. All of the previous studies utilizing the nylon fiber assay (2-5) have supported the concept that the intensity of adherence, measured with the nylon fiber columns, affects the ease of movement of granulocytes out of the vascular compartment; the present study directly confirms the concept that changes in adherence, observed with the nylon fiber assay, reflect adherence to endothelium.

When separated granulocytes are suspended in a medium devoid of plasma proteins, the adherence to endothelium and nylon remains similar; moreover, changes in adherence induced by cyclic nucleotides and pharmacologic agents are similar for both surfaces, and are equal when whole blood is used. Consequently, adherence to endothelium does not require plasma proteins or the presence of any other cell type, and modifications of adherence do not occur through the mediation of other cells. The higher degree of adherence that is noted when purified granulocytes are used in place of whole blood, may result from the exposure of cells to Hypaque-Ficoll, or the time required to process the cells.

Trypsinization has been used to remove proteincontaining components from cell membranes of many different cell types without killing them (16-18). Such treatment has been shown to remove as much as $15 \%$ of the dry weight of the cell (16), and $39 \%$ of its sialic acid content (17), while leaving $100 \%$ of the cells impermeable to trypan blue. In the present studies $95 \%$ of cells remained viable by trypan blue exclusion, and phase-contrast examination of the monolayer showed no visible abnormality after a 10-min exposure to various concentrations of trypsin. However, concentrations of $0.16 \%$ and greater caused major reduction of granulocyte adherence to the monolayers, suggesting that endothelium has a protein or glycoprotein substance on its membrane which promotes the attachment of granulocytes. This substance could be a specific receptor or perhaps a nonspecialized membrane component containing a charged moiety, such as sialic acid. The return of adherence to normal or increased levels by $24 \mathrm{~h}$ after trypsinization suggests that the substance is replaceable by the cell, even in the absence of exogenous serum proteins.

An alternative explanation for the action of trypsin on adherence is found in a recent report by Revel et al. (19) showing that trypsinization causes the monolayer cells to become round and lose their flattened appearance. A similar effect on endothelial monolayers would present a more difficult surface configuration for adherence of granulocytes, even though the monolayer remained intact and of normal appearance as seen using phase-contrast microscopy. Thereafter, the return to normal adherence characteristics could reflect a return of the cells to a flattened configuration rather than the regeneration of a surface component. Scamning electron microscopic study of the monolayer 
before and after trypsinization is necessary to evaluate these possibilities.

The availability of endothelial cells grown in monolayers now permits in vitro studies of the characteristics of granulocyte adherence to endothelium, and appears to represent an in vitro analog of granulocyte margination before diapedesis. The close correlation of adherence results on endothelial monolayers to those with nylon fiber columns, suggests that experiments using the nylon fiber assay system for granulocyte adherence are indicative of granulocyte-endothelium interactions in vitro; this cell-cell interaction in vitro may reflect events in the intravascular compartment.

\section{ACKNOWLEDGMENTS}

The authors thank Dr. Jaffe for his suggestions, the excellent technical assistance of Mrs. Elvira Ventura, Ms. Pamela Howard, Missy Cooper, and Terry Kirk, as well as Mrs. Kathleen Ludden's secretarial aid. Dr. Elias Abrutyn made helpful editorial suggestions.

This work was supported by U. S. Public Health Service Grants AA-00332, AM-14526, HL-15061, and HL-18827.

\section{REFERENCES}

1. Garvin, J. E. 1961. Factors affecting the adhesiveness of human leukocytes and platelets in vitro. J. Exp. Med. 114: $51-73$.

2. MacGregor, R. R., P. J. Spagnuolo, and A. L. Lentnek. 1974. Inhibition of granulocyte adherence by ethanol, prednisone, and aspirin, measured with a new assay system. N. Engl. J. Med. 291: 642-646.

3. Lentnek, A. L., A. D. Schreiber, and R. R. MacGregor. 1976. Induction of augmented granulocyte adherence by inflammation. Mediation by a plasma factor. J. Clin. Invest. 57: 1098-1103.

4. MacGregor, R. R. 1977. Granulocyte adherence changes induced by hemodialysis, endotoxin, epinephrine, and glucocorticoids. Ann. Intern. Med. 86: 35-39.

5. MacGregor, R. R. 1976. The effect of anti-inflammatory agents and inflammation on granulocyte adherence Evidence for regulation by plasma factors. Am. J. Med. 61: $597-607$.
6. Lewis, L. J., J. C. Hoak, R. D. Maca, and G. L. Fry. 1973. Replication of human endothelial cells in culture. Science (Wash. D. C.). 181: 453-454.

7. Jaffe, E. A., R. L. Nachman, C. G. Becker, and C. R. Minick. 1973. Culture of human endothelial cells derived from umbilical veins. Identification by morphologic and immunologic criteria. J. Clin. Invest. 52: $2745-2756$.

8. Gimbrone, M. A., R. S. Cotran, and J. Folkman. 1974. Human vascular endothelial cells in culture: growth and DNA synthesis. J. Cell Biol. 60: 673-684.

9. Macarak, E. J., B. V. Howard, and N. A. Kefalides. 1977. Properties of calf endothelial cells in culture. Lab. Invest. 36: 62-67.

10. Boyum, A. 1968. Isolation of mononuclear cells and granulocytes from human blood. Scand. J. Clin. Lab. Invest. 21(Suppl. 97): 77.

11. MacGregor, R. R. 1976. Cyclic nucleotide induction as a mechanism for modification of granulocyte adherence by plasma factors. Clin. Res. 24: 348.

12. Macarak, E. J., B. V. Howard, and N. A. Kefalides. 1976. Biosynthesis of collagen and metabolism of lipids by endothelial cells in culture. Ann. N. Y. Acad. Sci. 275: $104-113$.

13. Jaffe, E. A., L. W. Hoyer, and R. L. Nachman. 1973. Synthesis of antihemophilic factor antigen by cultured human endothelial cells. J. Clin. Invest. 52: 2757-2764.

14. Moscona, A. 1961. Effect of temperature on adhesion to glass and histogenic cohesion of dissociated cells. Nature (Lond.). 190: 408-409.

15. Marchesi, V. T., and H. W. Florey. 1960. Electron micrographic observations on the emigration of leukocytes. O. J. Exp. Physiol. Cogn. Med. Sci. 45: 343-348.

16. Codington, J. F., B. H. Sanford, and R. W. Jeanloz. 1970. Glycoprotein coat of the $\mathrm{TA}_{3}$ cell. I. Removal of carbohydrate and protein material from viable cells. J. Natl. Cancer Inst. 45: 637-647.

17. Snow, C., and A. Allen. 1970. The release of radioactive nucleic acids and mucoproteins by trypsinized and EDTA-treatment of baby hamster cells in tissue culture. Biochem. J. 119: 707-714.

18. Hughes, R. C. 1976. Membrane glycoproteins. In A Review of Structure and function. Butterworth \& Co. (Publishers) Ltd., London. 43-44.

19. Revel, J. P., P. Hoch, and D. Ho. 1974. Adhesion of culture cells to their substratum. Exp. Cell Res. 84: $207-218$. 